\title{
Software stereovision for optic laboratory
}

\section{Diego Henao}

Diego Alberto Ve Henao, "Software stereovision for optic laboratory," Proc. SPIE 2525, 1995 International Conference on Education in Optics, (13 October 1995); doi: 10.1117/12.224049

SDIE Event: SPIE's 1995 International Symposium on Optical Science, SPIE. Engineering, and Instrumentation, 1995, San Diego, CA, United States 
Software stereovision for optic laboratory

\title{
Diego Alberto Vélez Henao
}

Universidad Pontificia Bolivariana

Ingeniería Electrónica

\begin{abstract}
This paper surveys the basis of the stereoscopic chromatic systems which can be used to provide advanced three-dimensional physical simulations. Special attention is paid to the easy way for replacing unapproachable or high-priced methods like optical holography and high-tech stereoscopic lens systems. A computer program shows applications of stereoscopic procedures.
\end{abstract}

Key words: stereo vision, chromatic systems, three-dimensional physical simulations.

\section{GENERAL ASPECTS}

Stereoscopy is a phenomenon tied to our every day life and sensibility. Our relief conception about things as well as the nonconscius calculation of the distance that separates us from an object becomes possible due to our sophisticated sigth system.

Based on the physiological and psychologic steams, the relief vision has been explaied by different ways: the anatomist explains it under a physiological point of view, On the contrary, neurologist has a mental approach (processes of forming images on the brain).

This article wants to put into use a simple physical analysis which could be immdiately apply in computer programs. Thus physical simulations of three-dimensional phenomena could be obtained. In that way, some procedures demanding relief vision may be replaced - those sometimes are solved by using holography techniques -

\section{THREE - DIMENSIONAL VISION}

The stereopsis or stereovision is a phenomenon consisting of having a simultaneous vision with both eyes. Therefore, the visual sensation of third - dimension takes place.

Relief vision becomes possible because of our workable visual system. The hability to see in relief is owing to various factors as follows:

\subsection{Convergence}

The axes of the eyes are directed to the attention point forming a determined angle; that is called punctual vision. But when the outskirts are not directly focussed is called peripheral vision.

This could be explained by means of a diagram of rays: 


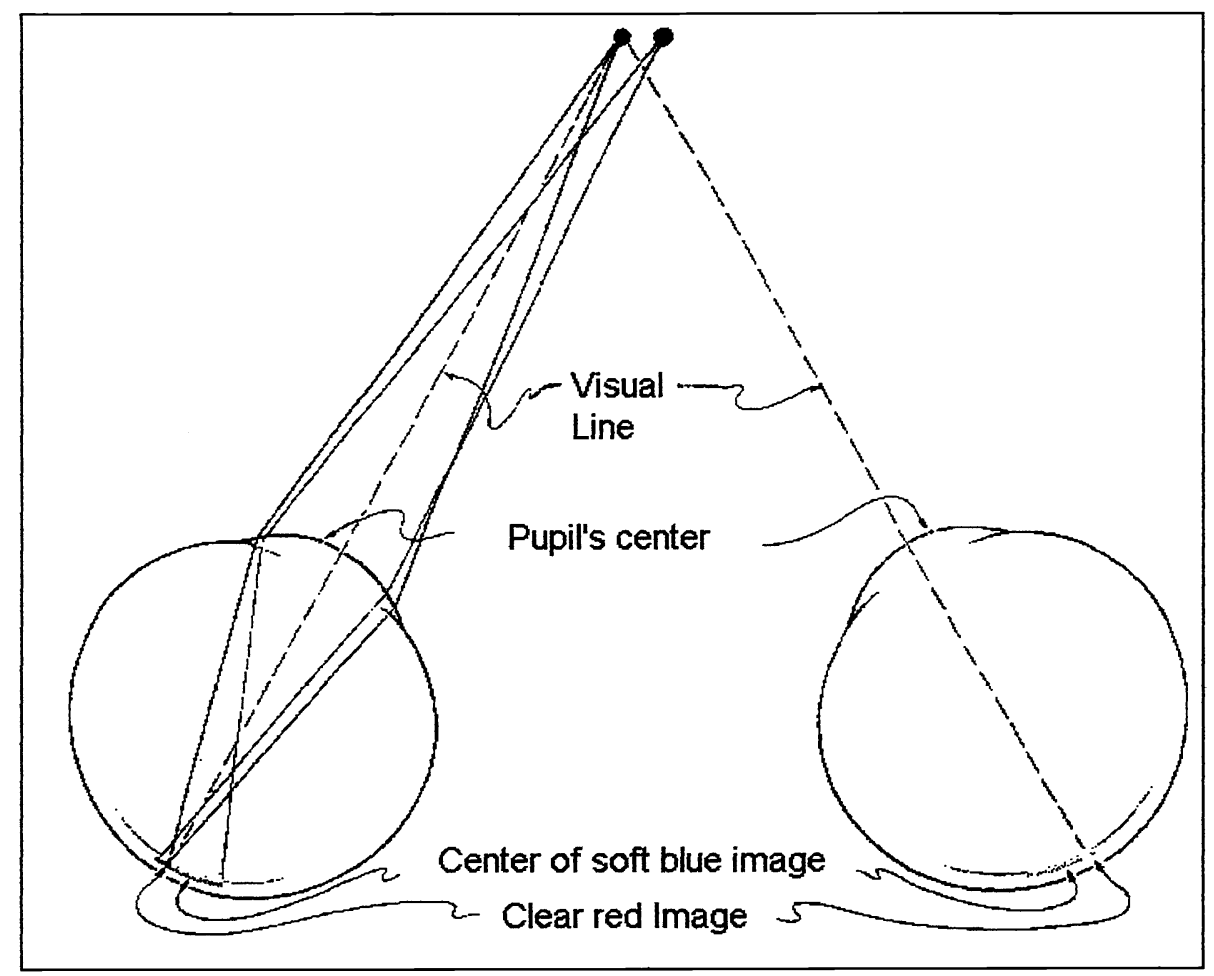

Fig. 1) Stereovision ray tracing

\subsection{Adaptation}

To take effect the focus, the distance between the retina and the crystalline lens must be variable. This distance is directly sent to the brain where it is processed in order to measure distances among objects.

\subsection{Light transformation in the nervous impressions}

Rays of light are turned into nervous impulses. Sensibility is proportional to the intensity (energy) of the lighting waves. Here, the light "strike" the cones and the rods; each of them are sensible to a determinated rank of frequency.

\subsection{Mental Projection}

Interpreting all the information given above is a brain task. All the information is transmitted by means of nervous ducts (this study belongs to the neurology). At the present time, it is believed that what we know as "outer world" is simply a model the brain had created.

\section{CHROMATIC STEREOSCOPY}

Stereovision through colors is easily obtained in a laboratory. For that purpose, it must decompose a photograpic image in two colors. It is necesary that their frequencies were too different. The most useful colors are blue and red. 
Images must have such a distance that the stereoscopic angle can be small ( 1 to 5 degrees). The observation of the stereoscopic figure is done by a pair of colored filters. On the one hand, if the element which is being visualized sends light out (additive synthesis) each filter allows the vision of the image at the same color. On the other, when talking in terms of subtrated sysnthesis, each filter blocks the same color compound, so it is possible to see the others.

Consequently, the rays take the same directions the eyes do when it is wanted to observe a three dimensional object. Thus, it is gotten an artificial sight of relief.

The absorption of a certain rank of wave length (color) in each filter resembles a experimental curve of the type:

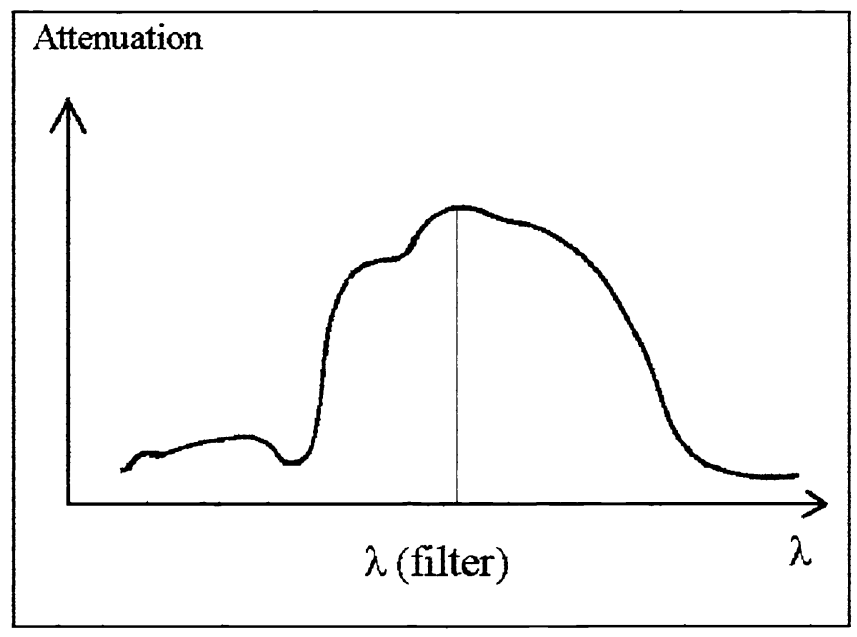

Fig. 2) Attenuation in plastic filter (subtrated sysnthesis)

\section{SOFTWARE STEREOVISION}

The relief vision could be used to enable effects in Computer Assistant Design Programs (CAD), physical simulations (fluxes, electromagnetic fields, collisions, properties of membranes, etc.) and three dimensional representations of atoms, molecules and compounds.

\subsection{Isometric projection}

It is practical to use algebraic models of projection when the object to be represented in the program can be described as a group of lines. Physical simulations often need movement, so it is better to use formulas which do not need so much calculations. In such way, the program is able to work faster. These formulas are obtained by means of geometric - descriptive procedures. 


$$
\begin{aligned}
& T: \Re^{3} \Rightarrow \Re^{2} \\
& \left(\begin{array}{l}
x_{i} \\
y_{i} \\
z_{i}
\end{array}\right) \rightarrow T\left(\begin{array}{l}
x_{i} \\
y_{i} \\
z_{i}
\end{array}\right)=\left(\begin{array}{l}
x_{p} \\
y_{p}
\end{array}\right)=\left(\begin{array}{c}
x_{c}+r_{p i x c l s}\left(\frac{y_{i}}{\operatorname{tam}_{y}} \sin (\theta)-\frac{x_{i}}{\operatorname{tam}_{x}} \cos (\theta)\right) \\
y_{c}-r_{p i x e l s}\left[\frac{x_{i}}{\operatorname{tam}} \sin (\theta) \cdot \cos (\phi)+\frac{y_{i}}{\operatorname{tam}_{y}} \cos (\theta) \cdot \cos (\phi)-\frac{z_{i}}{\operatorname{tam}_{z}} \cdot \sin (\phi)\right.
\end{array}\right)
\end{aligned}
$$

where:

$T$ : operator of projection.

$\left(x_{i}, y_{i}, z_{i}\right):$ three - dimensional coordinates of the point to be projected

$\theta, \phi:$ angles of observation, in the way they are defined by the spherical coordinates.

$r_{p i x e l s}$ : size constant for the figure in screen.

$\left(x_{p}, y_{p}\right)$ : screen coordinates of the projected point.

$\operatorname{tam}_{x}, \operatorname{tam}_{y}, \operatorname{tam}_{z}$ : factors of scale to each coordinate axis.

(Note that it is an isometric projection while it does not depend of the distance of observation.)

Using these equations, any $\mathrm{x}, \mathrm{y}, \mathrm{z}$ point is possible to be projected on a computer screen. These points may depend on a solid object or on a mathematics function (the former is helpful to make simulations of phenomena which are described by scalar o vector equations).

\subsection{Non - isometric projections}

To advanced simulations of dinamics of solids, it isoften used more complex models in which the object is oriented by using the angles of Euler as the figure shows it: 


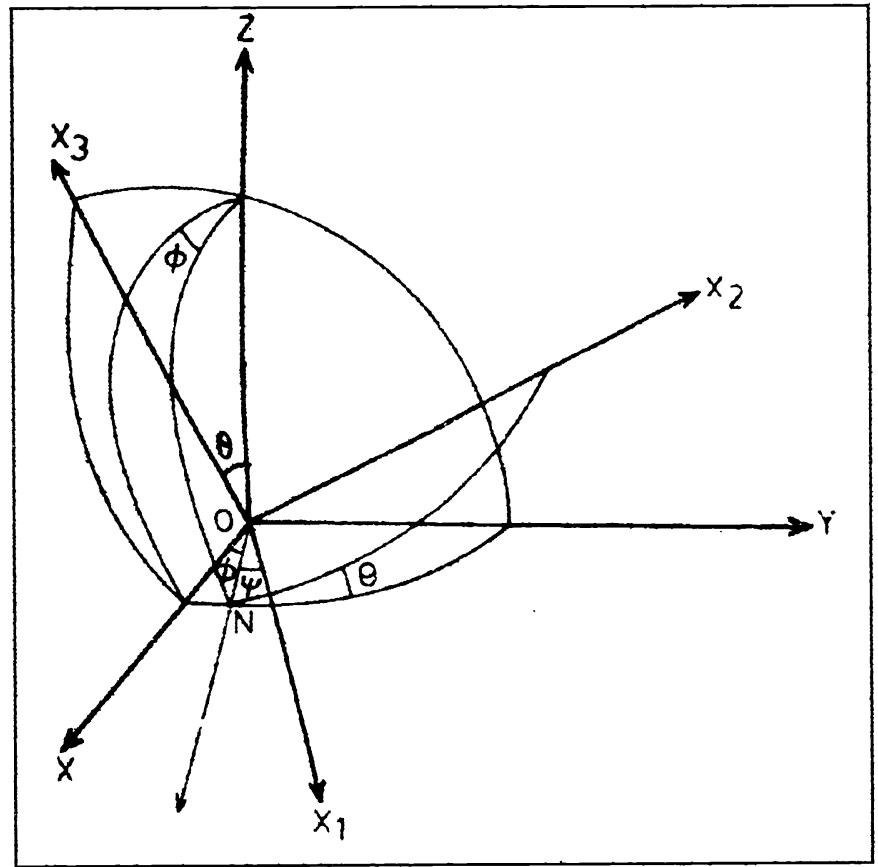

Fig. 3) The angles of Euler

A major realism is obtained by non - isometric projections based on the formation of images in the retina. The main idea is based on the geometric optics, as the figure shows:

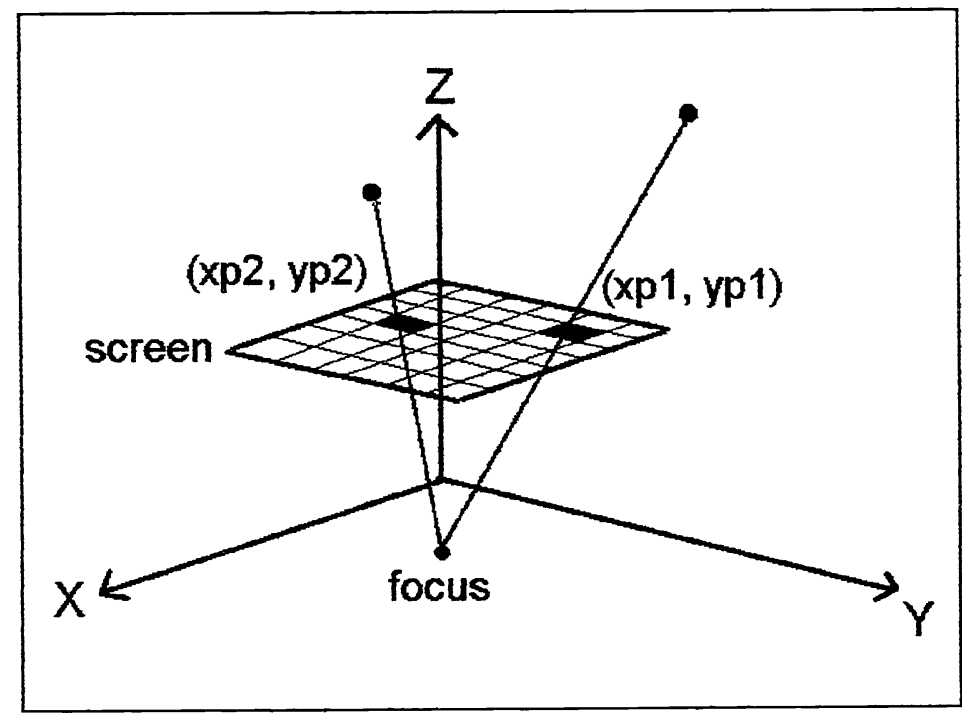

Fig. 4) No - Isometric proyection 
To reach the vision from any point out of the figure (location of the observation camera) rotation matrices are used. These "rotate" (make a mathematical transformation) the solid in the way it may be seen from the needed point.

The first rotation about the $Z$ axis can be described by a matrix $\mathbf{D}$,

$$
\mathbf{D}=\left(\begin{array}{ccc}
\cos \phi & \sin \phi & 0 \\
-\sin \phi & \cos \phi & 0 \\
0 & 0 & 1
\end{array}\right) \quad(2 . a)
$$

and the transformation equation can be written as

$$
\left(\begin{array}{l}
\xi \\
\eta \\
\zeta
\end{array}\right)=\mathbf{D}\left(\begin{array}{c}
X \\
Y \\
Z
\end{array}\right)
$$

Similary, as the second rotation is about the ${ }^{\xi}$ axis, the corresponding matrix $\mathbf{C}$ is given by

$$
\mathbf{C}=\left(\begin{array}{ccc}
1 & 0 & 0 \\
0 & \cos \theta & \sin \theta \\
0 & -\sin \theta & \cos \theta
\end{array}\right)
$$

with the transformation equation

$$
\left(\begin{array}{c}
\xi^{\prime} \\
\eta^{\prime} \\
\zeta^{\prime}
\end{array}\right)=\mathbf{C}\left(\begin{array}{l}
\xi \\
\eta \\
\zeta
\end{array}\right)(3 . b)
$$

For the final rotation, we writte the rotation matrix as

$$
\mathbf{B}=\left(\begin{array}{ccc}
\cos \psi & \sin \psi & 0 \\
-\sin \psi & \cos \psi & 0 \\
0 & 0 & 1
\end{array}\right) \quad \text { (4.a) }
$$

and the equation

$$
\left(\begin{array}{l}
X_{1} \\
X_{2} \\
X_{3}
\end{array}\right)=\mathbf{B}\left(\begin{array}{c}
\xi^{\prime} \\
\eta^{\prime} \\
\zeta^{\prime}
\end{array}\right)
$$

Combining Eqs. 2.b, 3.b and 4.b, we obtain

$$
\left(\begin{array}{l}
X_{1} \\
X_{2} \\
X_{3}
\end{array}\right)=\mathbf{B C D}\left(\begin{array}{l}
X \\
Y \\
Z
\end{array}\right)=\mathbf{A}\left(\begin{array}{l}
X \\
Y \\
Z
\end{array}\right)
$$

where $\mathbf{A}=\mathbf{B C D}$ 


$$
\begin{aligned}
\mathbf{A} & =\left(\begin{array}{ccc}
\cos \psi \cos \phi-\cos \theta \sin \phi \sin \psi & \cos \psi \sin \phi+\cos \theta \cos \phi \sin \psi & \sin \psi \sin \theta \\
-\sin \psi \cos \phi-\cos \theta \sin \phi \cos \psi & -\sin \psi \sin \phi+\cos \theta \cos \phi \cos \psi & \cos \psi \sin \theta \\
\sin \theta \sin \phi & -\sin \theta \cos \phi & \cos \theta
\end{array}\right) \\
\mathbf{A}^{-1} & =\left(\begin{array}{ccc}
\cos \psi \cos \phi-\cos \theta \sin \phi \sin \psi & -\sin \psi \cos \phi-\cos \theta \sin \phi \cos \psi & \sin \theta \sin \phi \\
\cos \psi \sin \phi+\cos \theta \cos \phi \sin \psi & -\sin \psi \sin \phi+\cos \theta \cos \phi \cos \psi & -\sin \theta \cos \phi \\
\sin \psi \sin \theta & \cos \psi \sin \theta & \cos \theta
\end{array}\right)
\end{aligned}
$$

This former matrix is used to get the $x, y, z$ coördinates beginning with a given coördinated vector in the rotated system. Each consecutive rotation corresponds with a matrixial product. It might verify that matrices are ortho-normal.

A great realism is gotten by orienting the solids with the angles of Euler projecting the points in a non isometric way and incorporating the stereovision.

The final result is given by the following algorithme:

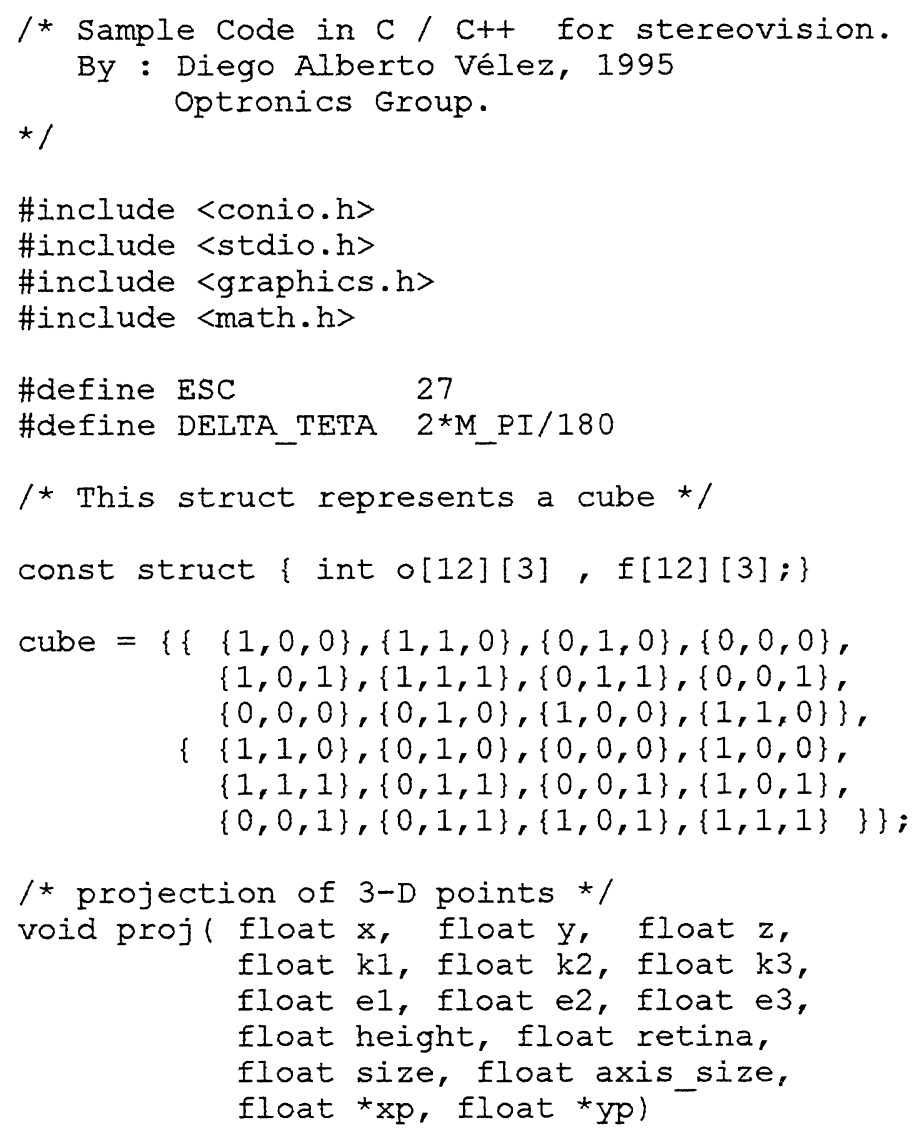




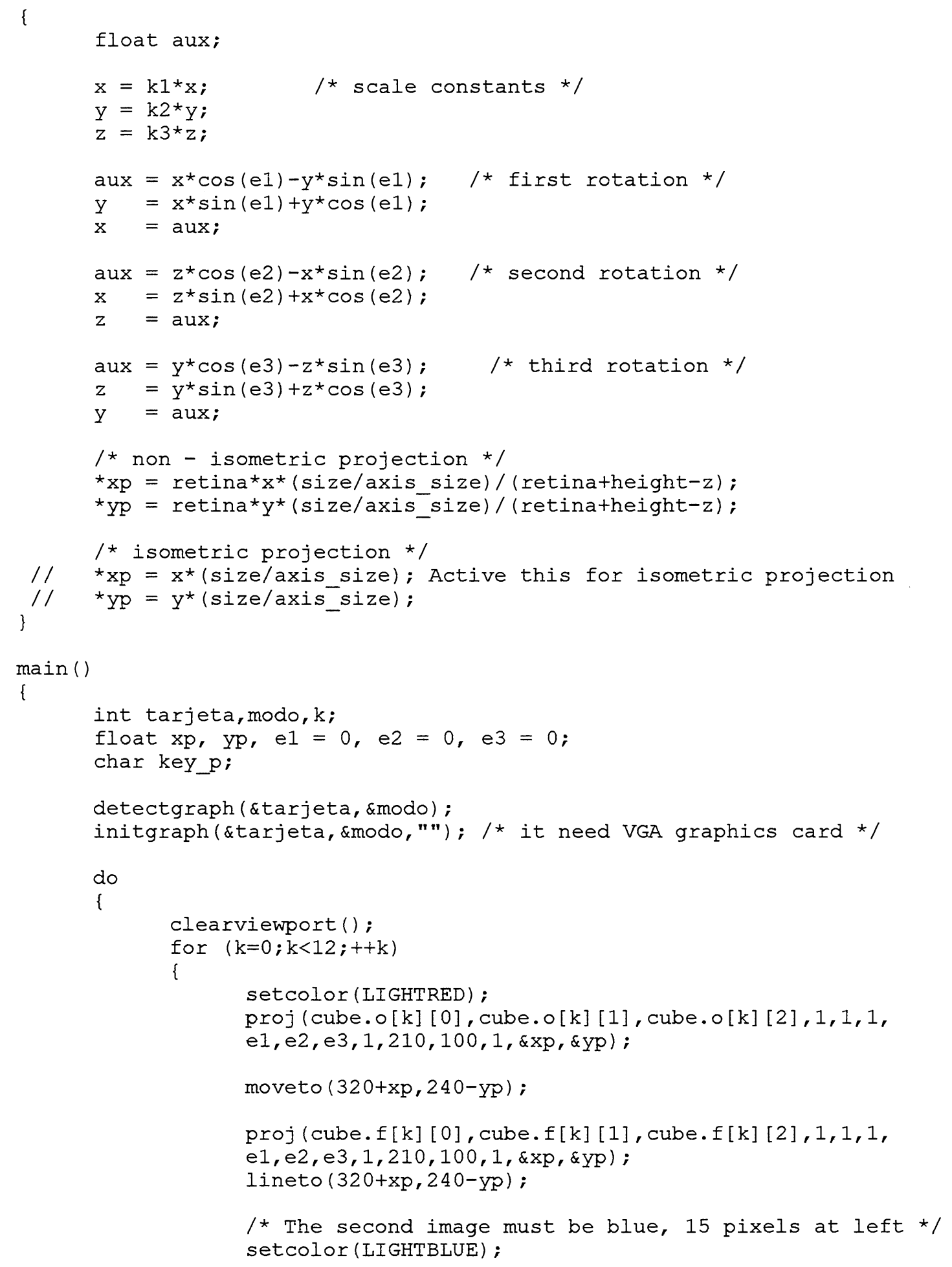




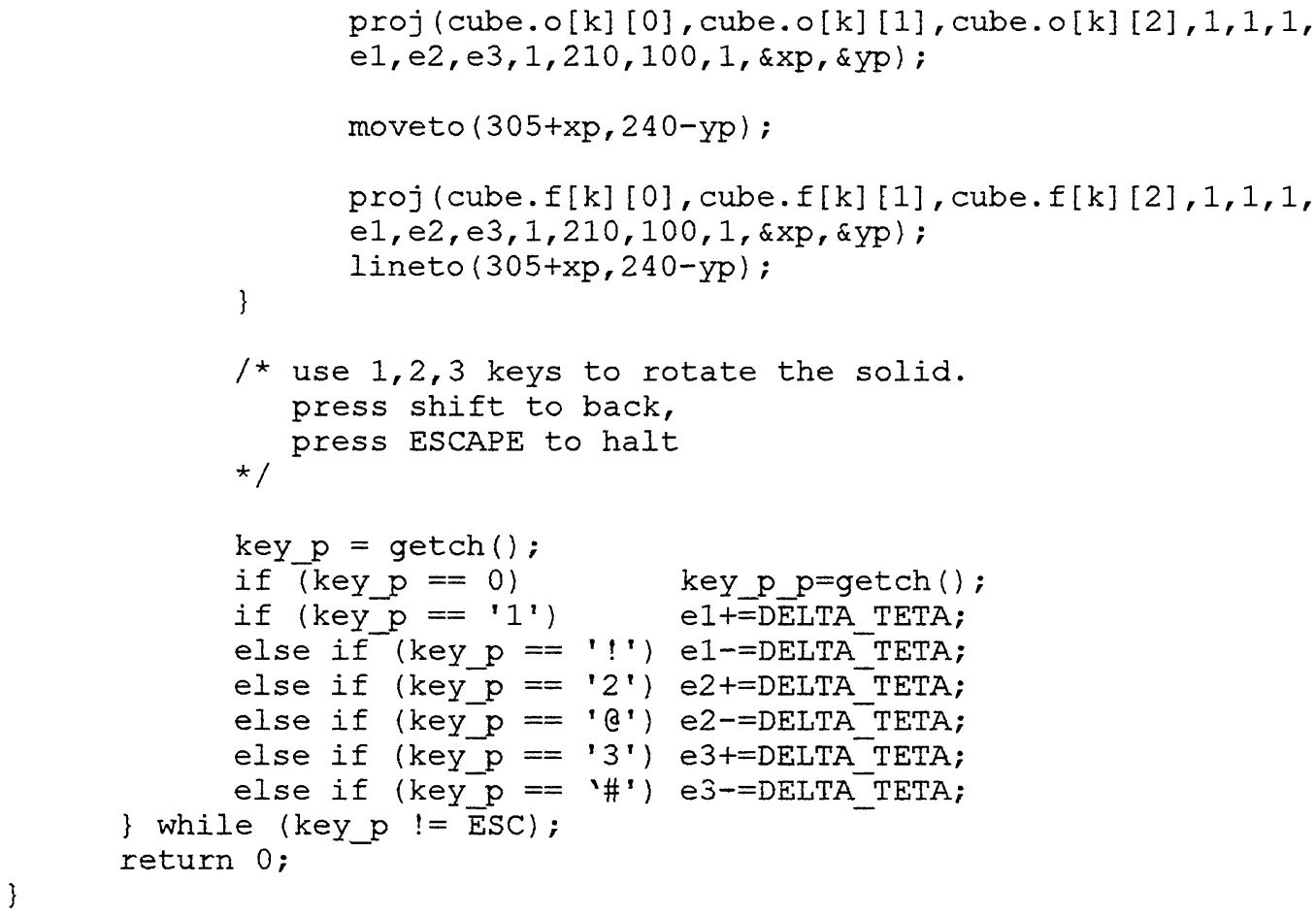

\section{CONCLUSIONS}

All phenomena of the stereoscopic vision can be explained by means of the geometric optics using a simple diagram of rays. This is enough to incorporate a major realism to the physics simulations through a three dimensional event computer.

The relief vision becomes possible to human beings because of four (4) factors which provide the brain:

1. Convergence of the eyes axes (punctual vision).

2. Distance adaptation between the crystalline lens and the retina.

3. Nervous impulses which come from the lighting sensation (this is called flat vision).

4. Mental projection or re - created model by the brain.

Equations of projection allow to show on the screen three - dimensional objects. Its application field could take effect in topography, architectonic and mechanic design, physics simulations, atomic, molecular or compounds models, and any other application which need a sensation of relief. In that way, It is possible to figure out correctly that stereovision does not belong to an specific field.

Computing gives a tool for making chromatic stereoscopy without photography, while it makes room to the movement ruled by mathematics expressions, visualization of surfaces, lines, fields, etc. in a common computer monitor and in a lower price too.

Advances could be greater to the extent that technology improves all the means of visualization in cathode ray tubes (or liquid crystal screens) as well as it supplies a greatter rate to the computer. 
Simulation by means of stereovision is placed as a proposal as well as the question that it could exist a via of visualization that emits polarized light in a variable angle. All of that, may increase quuality by replacing the colored filters with polaroids which must be displaced from each other 90 degrees.

Every day, the science gives us more and more tools and is our work to make use of them in the best way, so that we may be the real participants in the new technologic paths.

\section{ACKNOWLEDGMENTS}

Grateful acknowledgement to La Universidad Pontificia Bolivariana, Medellín, Colombia for making possible this work.

Professor Samuel Angel Jaramillo Flórez who guided this work and helped with opportune remarks and suggestions.

\section{REFERENCES}

1. H. C. Raasveld, "Investigación de la visión estereoscópica", Revista de la Academia Colombiana de Ciencias Exactas, Físicas y Naturales. pp 288-314. 1956.

2. Walker, Jearl, "El hiperescopio y el pseudoscopio facilitan la experimentación sobre la visión tridimensional". Revista Investigación y Ciencia. Vol. 124, pp 94-98. October 1982.

3. Grabulosa, Joan Batlle y otros, "Interpretación Físico-Matemática del color". Revista Española de Electrónica. pp 20-27. October 1993.

4. Brou, Phillippe y otros, "Los colores de las cosas". Revista Investigación y Ciencia. Vol. 123. pp 56-64. September 1982.

5. Fletcher, Alvaro González, "La estéreoortofotografia en la elaboración de mapas". Revista de la Academia Colombiana de Ciencias Exáctas, Físicas y Naturales. pp 73-89. 1986

6. Bulabois, Jean and Tribillon, Gilbert. "Las imágenes en tres dimensiones". Revista Mundo Científico. Vol 27. pp 732-745. 1983. 